The Egyptian Journal of Hospital Medicine (July 2019) Vol. 76 (2), Page 3589-3594

\title{
Intralesional Vitamin D3 versus Purified Protein Derivatives in The Treatment of Multiple Cutaneous Warts: Comparative Study Essam Elden M Mohamed ${ }^{a}$, Moustafa A El Taieb ${ }^{b}$, Gehad A Abd El- sabour ${ }^{b}$ \\ a. Venereology and Andrology, Faculty of Medicine, Al-Azhar University, Assuit, \\ b. Dermatology, Venereology and Andrology, Faculty of Medicine, Aswan University \\ Corresponding author: Gehad A Abd El- sabour,email: gehada022@gmail.com
}

\begin{abstract}
Background: Viral warts are caused by human papillomavirus. Many therapeutic options are available for treating warts. Newer and effective treatments include intralesional immunotherapy, has been tried in the recent times with variable success rates. Objective: The aim of this study was to compare the effect of intralesional vitamin D3 versus purified protein derivative (PPD) in the treatment of multiple cutaneous warts.

Methods: A total of 62 patients were included in the study, each patient had at least 3 common warts, one or more of them injected with 0.1 of intralesional PPD, one or more injected with 0.5 of vitamin D3 and one or more were control according to number of warts of each patient and up to 4 sessions. The clinical assessment was done by photographic measurements at baseline, before each treatment session, and after the completion of treatment.

Results: In PPD-injected lesions. 43 patients (69.4\%) showed complete clearance (100\%) and 13 patients (20.9\%) showed partial response (25\% - 99\%). While, in vitamin D3-injected lesions, 22 patients (35.5\%) showed complete clearance while 21 patients (33.9\%) showed partial response. On comparing between PPD, vitamin D3 and control, both PPD and vit D3 were statistically significant compared to control and PPD was more statistically significant than vit D3 (P3 = 0.019) in effectiveness and less in recurrence rate. Conclusions: PPD and vitamin D3 were found to be effective, well-tolerated modalities of treatment of multiple common warts. Intralesional PPD was more statistically significant in effectiveness and less in recurrence rate than intralesional vitamin D3.
\end{abstract}

Keywords: Intralesional, PPD, cutaneous wart, vitamin D3.

\section{INTRODUCTION}

Warts are common, benign and self-limiting epidermal proliferations or tumors of the skin and mucosa caused by HPVs ${ }^{(1)}$. The prevalence of HPV infection was estimated to be $7-12 \%{ }^{(2)}$.About two thirds of warts regress spontaneously within 2 years ${ }^{(3)}$. Many therapeutic options are available for treating warts, with none is uniformly effective ${ }^{(4,5)}$. The warts were treated by variable methods, such as topical agents, cryosurgery, electrocautery and laser ablation $(6,7)$. Classical treatment lines are limited to local application and do not act systemically ${ }^{(5)}$. Immunotherapy depends on the ability of the immune system to recognize bacterial, viral and fungal antigen that induce a delayedtype hypersensitivity response to different antigens and also the wart tissue, which induce the ability of the immune system to recognize and eradicate HPVs ${ }^{\left({ }^{(8)}\right.}$.

Immunotherapy is indicated in recalcitrant warts, recurrent warts and extensive warts and difficult to treat areas as periungual and palmoplantar sites ${ }^{(9)}$. Many agent of immunotherapy that have been tried include cimetidine, imiquimod, interferons, Candida albicans antigens, measles, mumps, rubella (MMR) vaccine, purified protein derivative (PPD) and intralesional vitamin D3 ${ }^{(\mathbf{1 0 , 1 1})}$.

Intralesional PPD and vitamin D3 are effective and recent modalities for the treatment of warts. So we undertook a study to compare the safety and efficacy of intralesional immunotherapy using PPD and vitamin D3 in the treatment of multiple viral warts.

\section{METHODS}

A total 62 patients ( 28 males and 34 female), aged 6 to 56 years, from Outpatient Clinic of Dermatology, Venereology and Andrology, Aswan University Hospital, Aswan University, Egypt were included in this study in the period between October 2017 and March 2018. The study was approved by the Local Institutional Ethics Committee of Faculty of Medicine, Aswan University. All participants were informed about the nature of the study, and written informed consent was obtained.

Patients with multiple common cutaneous warts and with a past history of BCG vaccination were included in this study. Immunocompromised patients as cancer and HIV patients, pregnant and lactating females, patients with history of hypersensitivity to vitamin D3, patients with past history of tuberculosis or negative tuberculin test and patients received any warts treatment 1 month before the start of the study, were excluded from this study.

Each patient was assessed by detailed history, general examination, and local examination, photographing the patient at baseline and every 2 weeks after the procedure was performed and follow up of the patient for 3 months to detect recurrence. All patients participated in the study had at least three warts. One or 
more of them were injected with PPD, one or more were injected with vitamin $\mathrm{D}$ and one or more were control according to each patient and number of warts.

\section{Treatment Protocols:}

Intralesional

PPD

was injected using a dose of $10 \mathrm{IU}(0.1 \mathrm{ml})$ with an insulin syringe in the selected wart. A $2 \mathrm{ml}$ vial of PPD (Mantoux test), which is an intradermal test (manufactured by Vacsera Company, Cairo, Egypt) was used. The product was stored in the dark, except when doses are actually being withdrawn from the vial between 2 and $8{ }^{\circ} \mathrm{C}$. The expiry date of a vial of tuberculin PPD is 1 month from the date of opening it (12). Vitamin D3 for injection is available in vials containing 200,000 IU of cholecalciferol in $2 \mathrm{ml}$ (manufactured by CHEMIPHARM Pharmaceuticals Industries, Cairo, Egypt). The selected warts were slowly intralesional injected with $0.5 \mathrm{ml}$ vitamin D3 solution into the base of the wart with a 27 -gauge insulin syringe.

Both intralesional PPD and vitamin D3 were injected at regular interval of 2 week until resolution or for a maximum of 4 treatment sessions. Assessment of treatment efficacy took place based on clinical examination and photography evaluation using Nikon Coolpix S 2500 camera 12 MP. Results were assessed at end of 8 weeks.

The results were assessed as:

- Excellent response: responders who showed 100\% clearance.

- Very good response: responders who showed 75 99\% clearance.

- Good response: responder who showed 50 - 74\% clearance.

- Poor response: responder who showed 25 - 49\% clearance

- $\quad$ No response: responder who showed $0-24 \%$

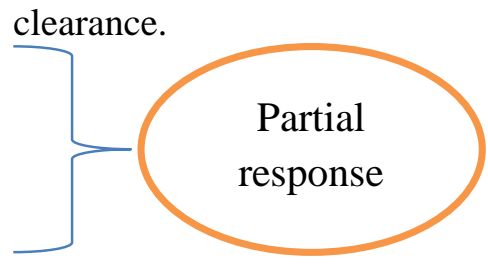

\section{Statistical analysis}

Data were verified, coded by the researcher and analyzed using IBM-SPSS version 21.0. Descriptive statistics: Means, standard deviations, medians, ranges and percentages were calculated. Test of significances: chi-square test was used to compare the difference in distribution of frequencies among different groups. For continuous variables; independent t-test analysis was carried out to compare the means of normally distributed data. A significant $\mathrm{p}$ value was considered when it is equal or less than 0.05 .

\section{RESULTS}

62 patients with multiple common warts were enrolled in this study. 28 patients $(45.2 \%)$ were males and 34 patients (54.8) were females. The age of the patients ranged from 6 to 56 years old with mean ages $25.61 \pm$ 10.7 years. The duration of the lesions ranged from 3 month to 24 months with mean duration $7.65 \pm 0.6$ months. According to the site of the lesion, 8 patients (12.9\%) had lesions in head and neck, 48 patients (77.4\%) had lesions in upper limb and 6 patients (9.7\%) had lesions in lower limb.

In the PPD-injected lesions, the complete clearance $(100 \%)$ was observed in 43 patients $(69.4 \%)$, Partial response $(25 \%-99 \%)$ was observed in 13 patients $(20.9 \%)$ and no response $(0-24 \%)$ was observed in 6 patients $(9.7 \%)$. In the vitamin D3-injected lesions, complete improvement was observed in 22 patients $(35.5 \%)$, partial response was observed in 21 patients $(33.9 \%)$ and no response was observed in 19 patients $(30.6 \%)$. In the control lesions, the complete clearance was observed in 12 patients (19.4\%), partial response was observed in 9 patients $(14.5 \%)$ and no response was observed in 41 patients $(66.1 \%)$ (Figure 2,3$)$.

On comparing between PPD, vitamin D3 and control, both PPD and vitamin D3 were statistically highly significant than control that, P1 (between PPD and control) was $<0.001$ and P2 (between control and it D3) was 0.026. PPD was statistically significant than vit D3, that, P3 between them was 0.019 (Table 1).

With regard to comparison of complications, pain at the site of injection was the most common complications with both modalities and significant with PPD, swelling at the site of injection was more with vitamin D3 than PPD, recurrence and other complication as eczema, pigmentation and inflammation were more with vitamin D3 (Figure 1)

Regarding the predictors for successful improvement after treatment with PPD injection, there was a significant correlation between improvement and disease duration, number of sessions and swelling after injection. There was no correlation between improvement and age, sex, site of the lesions and other complications. By multivariate regression analysis, we found that the most significant factors were the disease duration and number of sessions (Table 2).

According to the predictors for successful improvement after treatment with vitamin D3, there was a significant correlation between improvement and sex. Males gave better results than females. Disease duration, number of session, lesions site, which in upper limb correlate with improvement of the swelling after injection and the pain. There was no correlation between improvement and age. By multivariate regression analysis, we found that the most significant factors were 
ejhm.journals.ekb.eg

sex, disease duration, number of session and swelling

(Table 3).

Table 1: Comparative analysis between the studied groups in improvement

\begin{tabular}{|l|l|l|l|l|l|l|}
\hline Improvement & Control & PPD & Vit-D & P-value* & P3 $=0.019$ \\
\hline Good-Excellent & $20(32.3 \%)$ & $54(87.1 \%)$ & $37(59.7 \%)$ & P1<0.001 & P2=0.026 & P3=0.01\%) \\
\cline { 1 - 4 } $\begin{array}{l}\text { Poor-No } \\
\text { Response }\end{array}$ & $42(67.7 \%)$ & $8(12.9 \%)$ & $25(40.3 \%)$ & & & \\
\hline
\end{tabular}

*Chi-square test was used to compare the percentages between groups

$\mathrm{P} 1=$ Control vs. PPD

$\mathrm{P} 2=$ Control vs. Vit-D

P3=PPD vs Vit-D

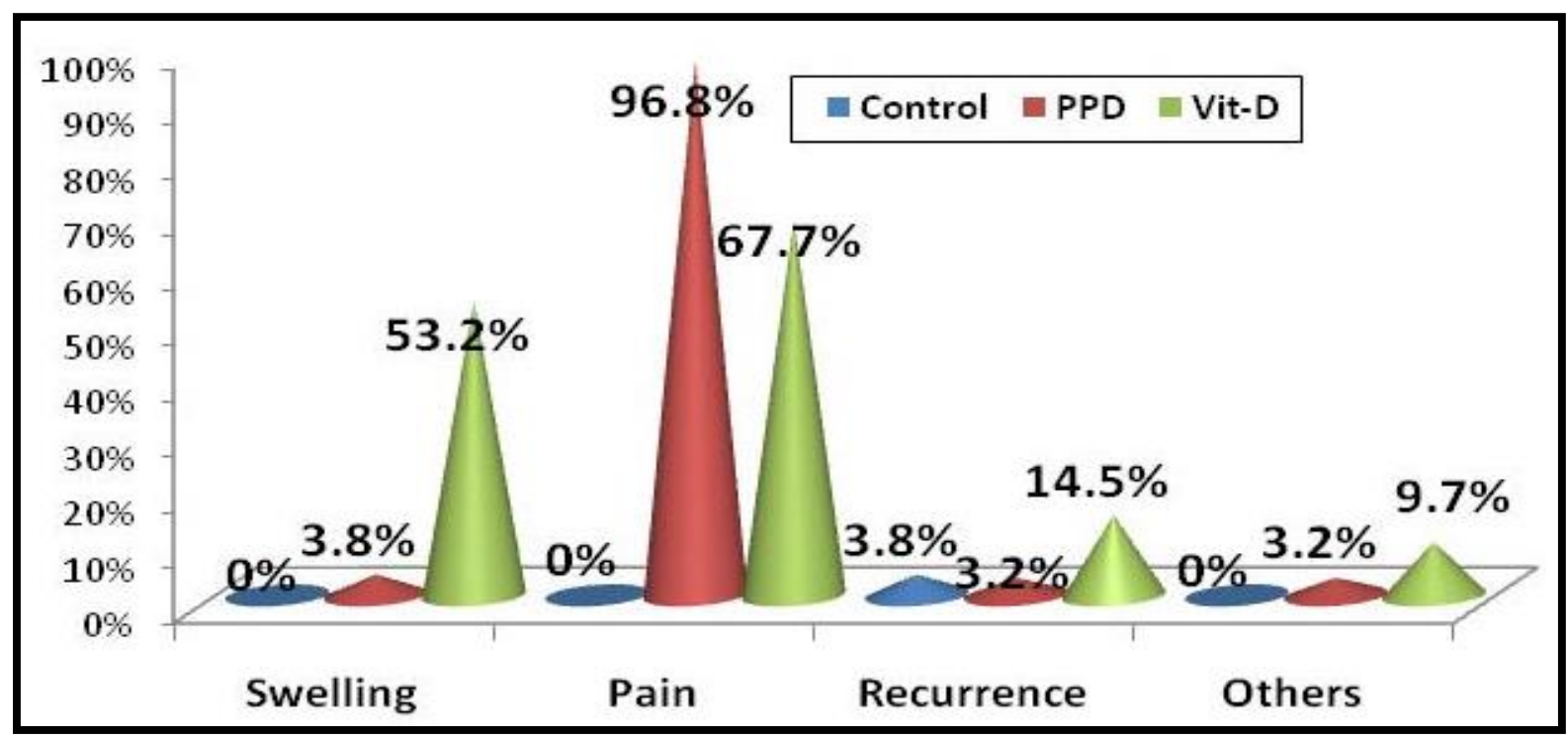

Figure (1): Prevalence of Complications among Different Treatment samples

Table (2): Correlation Coefficient between clinical parameter and improvement after PPD injection: Multivariate Regression Analysis

\begin{tabular}{|l|c|c|c|}
\hline Variable & Odds Ratio & 95\% CI* & LRT** P-value \\
\hline Age & 1.017 & $0.95-1.10$ & $=0.647$ \\
\hline Sex (Male) & 2.786 & $0.52-15.05$ & $=0.234$ \\
\hline Disease Duration in months & $\mathbf{3 . 6 0 1}$ & $\mathbf{1 . 4 2}-\mathbf{7 . 5 6}$ & $=\mathbf{0 . 0 1 2}$ \\
\hline No. of Sessions & $\mathbf{1 . 2 4 4}$ & $\mathbf{1 . 0 1}-\mathbf{2 . 6 1}$ & $=\mathbf{0 . 0 2 3}$ \\
\hline
\end{tabular}

Table (3): Correlation Coefficient between clinical parameter and improvement after vit D3 injection: Multivariate Regression Analysis

\begin{tabular}{|l|c|c|c|}
\hline Factor & $\begin{array}{c}\text { Odds } \\
\text { Ratio }\end{array}$ & $\mathbf{9 5 \%}$ CI* $^{*}$ & LRT** P-value \\
\hline Age & 1.034 & $0.98-1.09$ & $=0.201$ \\
\hline Sex (Male) & $\mathbf{3 . 3 7 5}$ & $\mathbf{1 . 1 4}-\mathbf{1 0 . 0 3}$ & $=\mathbf{0 . 0 2 9}$ \\
\hline Disease Duration in months & $\mathbf{1 . 6 2 4}$ & $\mathbf{1 . 2 5}-\mathbf{2 . 1 1}$ & $<\mathbf{0 . 0 0 1}$ \\
\hline No. of Sessions & $\mathbf{1 . 0 7 2}$ & $\mathbf{1 . 0 1}-\mathbf{3 . 2 9}$ & $=\mathbf{0 . 0 3 4}$ \\
\hline
\end{tabular}


ejhm.journals.ekb.eg

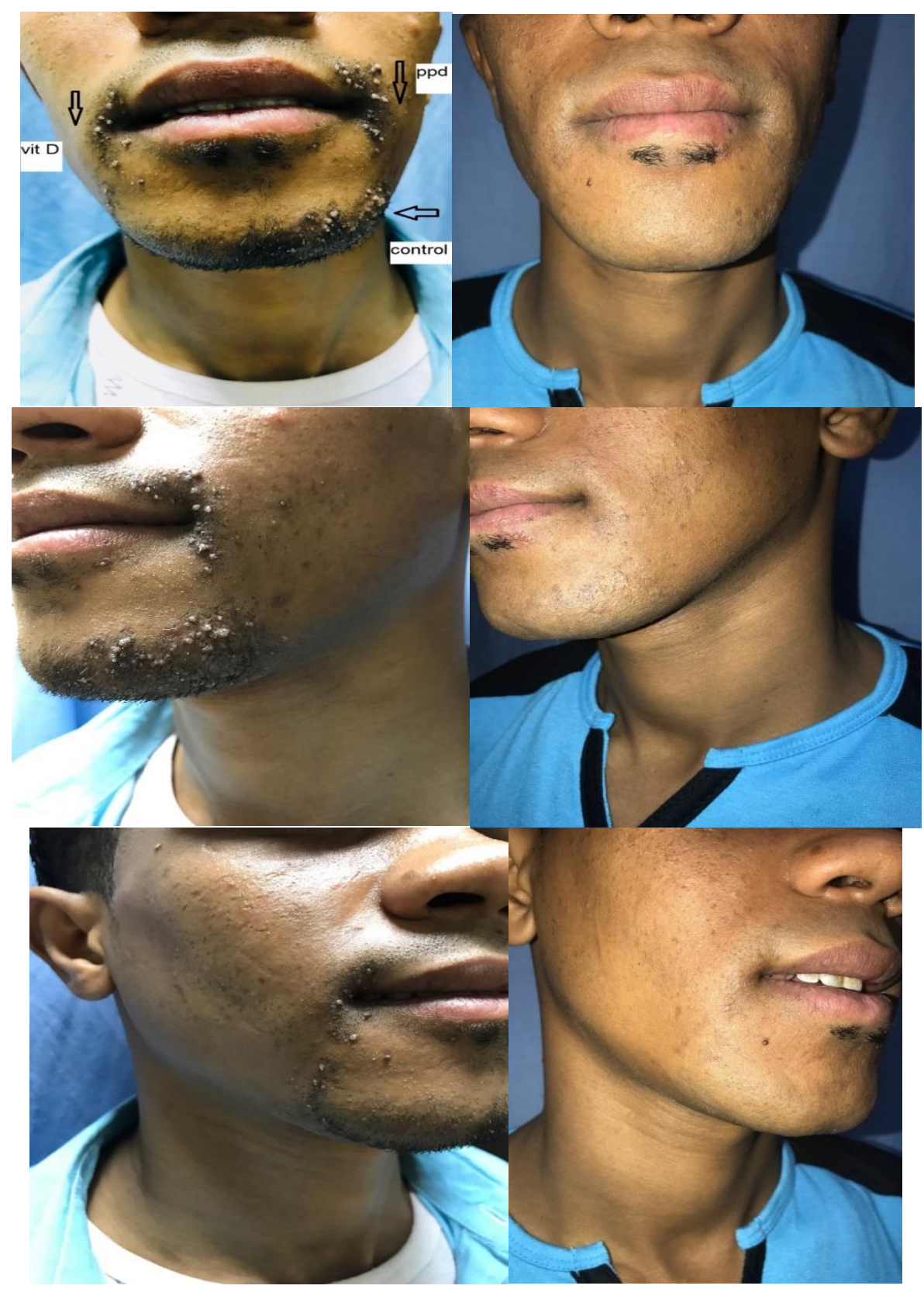

Figure (2): Complete clearance of PPD, vit D3and control after second session
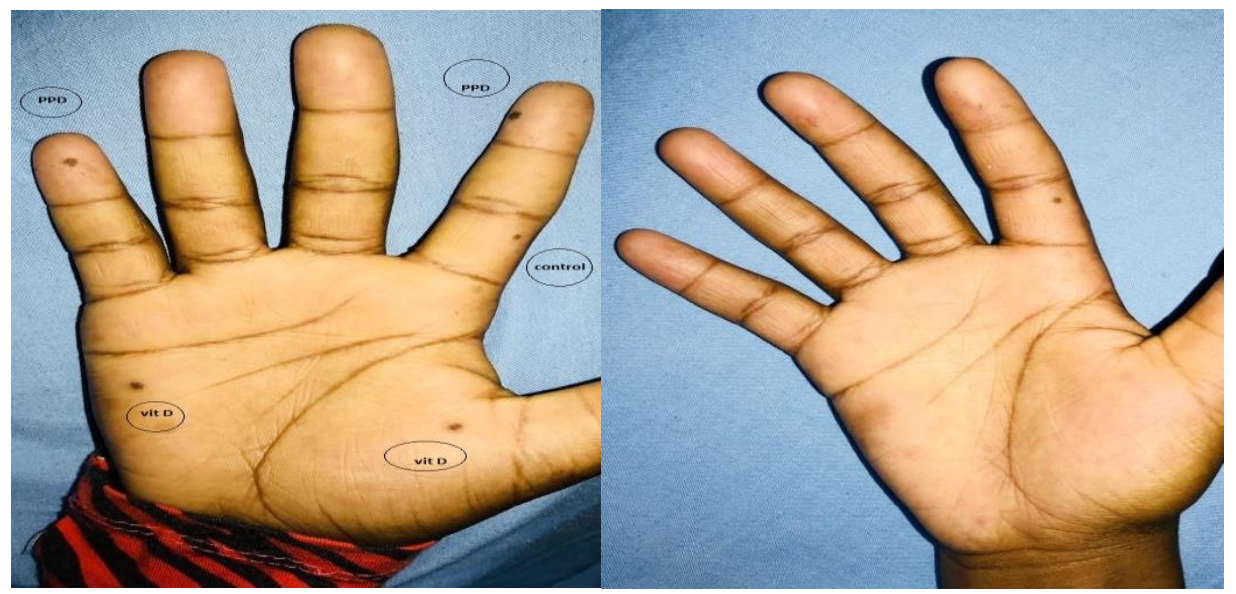
Figure (3): Before and after third session

\section{DISCUSSION}

Intralesional PPD generates strong proinflammatory signals and attracts antigenpresenting cells, which recognize HPV particles in the infected tissue leading to a strong adaptive immune response that helps in clearing the infection. PPD has been found to be associated with the production of Th1 cytokines such as IL4, IL-5, IL-8, IL-12, INF $\gamma$ and TNF $\alpha$, which motivate cytotoxic and NK cells that stimulate a strong immune response against HPV ${ }^{(13,14,15,16)}$.

Regarding the intralesional PPD, our result is close to match with results reported by Saoji $\boldsymbol{e t}$ al. that showed out of 55 patients, $42(76 \%)$ patients showed complete clearance after four sessions while $13(24 \%)$ patients were non-responders (No or partial clearance of the lesions) ${ }^{(17)}$. Results achieved by Singh $\boldsymbol{e t}$ al. on 40 patients, 32 (80\%) patients had a complete clearance, 6 patients $(15 \%)$ had partial clearance and 2 patients $(5 \%)$ had no improvement. The different in clearance rate may be explained by the differences in the number of patients, the duration, the types and the resistance of warts ${ }^{(\mathbf{1 6})}$.

There was a significant correlation between duration of disease and the improvement, which means longer duration of the disease, the more response to PPD. This may be because younger warts represent a higher viral amount when compared to old warts ${ }^{(\mathbf{1 8})}$. This does not match with the results obtained by Kus et al. who reported that the response to PPD injection is not affected by the duration of the lesions ${ }^{(19)}$, also does not match with Elela $\boldsymbol{e t}$ al. who reported that the response to PPD injection is affected by the duration of the lesions, the longer the duration the less the response to PPD ${ }^{(20)}$.

Vitamin D3 regulates epidermal cell proliferation and differentiation and modulates cytokine production. It acts on activation of Tolllike receptor which causes human macrophages up-regulation and expression of VDR and VD1hydroxylase genes, leading to production of the antimicrobial peptide ${ }^{(7,5)}$. The results achieved by Singh et al. on 40 patients, 29 patients (72.5\%) had a complete response, 8 patients $(20 \%)$ had partial response and 3 patients $(7.5 \%)$ had no response ${ }^{(16)}$. A study done by Raghukumar et al. on 60 patients showed that 54 patients $(90 \%)$ showed complete clearance while 4 patients
(6.66\%) showed partial response and 2 patients (3.33\%) showed no response ${ }^{(5)}$.

The clearance rates in our study were different from the previous studies, which can be explained by the changes in the number of the patients, type of the wart and the concentration of vitamin D3. In previous studies, they used vitamin D3 solution $(600,000 \mathrm{IU}, 15 \mathrm{mg} / \mathrm{ml})$ but in our study, we used vitamin D3 solution (200,000 IU).

There was a significant correlation between improvement and sex, which means that improvement, was better in male than female. Also there was significant correlation between improvement and number of session, duration of disease and swelling at site of injection, which may be due to induced reaction. By multivariate regression analysis, the most important factors affected in improvement were sex of the patient, duration of the disease and number of the sessions.

Complications of both modalities included, pain at the site of injection was the most common with both modalities; swelling at the site of injection was more significant with vitamin D3 than PPD; other complication as eczema, pigmentation and inflammation were more with vitamin D3. Recurrence occurred in 9 cases with vitamin D3 while occurred in 2 cases with PPD.

In control lesions, there were response occurred in 21 patients and no response in 41patients. The response that occurred may be explained by spontaneous regression of warts as without treatment, approximately $23 \%$ of the lesions show spontaneous regression in 2 months, $30 \%$ spontaneously regresses in 3 months and $65 \%$ to $78 \%$ regress spontaneously in 2 years ${ }^{(21)}$, or it may be due to systemic effect of PPD, in which the immune response not restricted to site of the injection ${ }^{\mathbf{2 0}, \mathbf{2 2})}$. We obtained good results by both modalities. Overall, intralesional PPD was superior to intralesional vitamin D3. There was a statistically significant difference in results between both modalities and this match with the results previously achieved by Singh et al. ${ }^{(16)}$.

\section{CONCLUSION}

Intralesional PPD was more effective than intralesional vitamin D3 in the treatment of cutaneous common wart and less in recurrence rate than vitamin D3. It is simple to perform, not very painful, inexpensive, effective and safe 
procedure with no residual scars. It may represent an alternative treatment for multiple common warts.

\section{Limitations:}

There was variability in the size of the warts.

\section{REFERENCES}

1. Sterling JC, Gibbs S, Haque Hussain SS, Mohd Mustapa MF, Handfield-Jones SE (2014): British association of dermatologists' guidelines for the management of cutaneous warts 2014.British Journal of Dermatology., 171(4):696-712.

2. Lynch M, Cliffe J, Morris-Jones $R$ (2014): Management of cutaneous viral warts. BMJ., 348:g3339.

3. Mun JH, Kim SH, Jung DS, Ko HC, Kim BS, Kwon KS, Kim MB (2011): Oral zinc sulfate treatment for viral warts: an open-label study. Journal of Dermatology, 38(6):541-545.

4. Mulhem E, Pinelis S (2011): Treatment of nongenital cutaneous warts. Am Fam Physician, 84(3):288-93.

5. Raghukumar S, Ravikumar BC, Vinay KN, Suresh MR, Aggarwal A, Yashovardhan DP (2017): Intralesional Vitamin D3 injection in treatment of recalcitrant warts: a novel proposition. J Cutan Med Surg., 21(4):320-4. 29.

6. Choi JW, Cho S, Lee JH (2011): Does immunotherapy of viral warts provide beneficial effects when it is combined with conventional therapy? Ann Dermatol., 23 (3): 282-7.

7. Aktaş H, Ergin C, Demir B, Ekiz O (2016): Intralesional vitamin $\mathrm{D}$ injection may be an effective treatment option for warts. J Cutan Med Surg., 20: 118122.

8. Nofal A, Salah E, Nofal E, Yosef A (2013): Intralesional antigen immunotherapy for the treatment of warts: current concepts and future prospects. Am J Clic Dermatol., 14:253-260.

9. Thappa DM, Chiramel MJ (2016): Evolving role of immunotherapy in the treatment of refractory warts. Indian Dermatol Online J., 7(5):364370.

10. Majid I, Imran S (2013): Immunotherapy with intralesional Candida albicans antigen in resistant or recurrent warts: A study. Indian J Dermatol., 58:360-5.
11. Meena JK, Malhotra AK, Mathur DK, Mathur DC (2013): Intralesional immunotherapy with Mycobacterium $\mathrm{w}$ vaccine in patients with multiple cutaneous warts: Uncontrolled open study. JAMA Dermatol., 149:237-9.

12. Landi S, Held HR (1986): Effect of oxidation on the stability of tuberculin purified protein derivative (PPD). Dev Biol Stand., 58 (Pt. B):545-52.

13. Gupta S, Malhotra AK, Verma KK, Sharma VK (2008): Intralesional immunotherapy with killed Mycobacterium w vaccine for the treatment of ano- genital warts: An open label pilot study. J Eur Acad Dermatol Venereal., 22:1089- 93

14.Abd-Elazeim FMA, Mohammed GFA, Fathy A, Mohamed RW (2014): Evaluations of IL12 serum level in patients with recalcitrant multiple common warts, treated by intralesional tuberculin antigen. $\mathrm{J}$ Dermatol Treat., 25: 264-7.

15. Nimbalkar A, Pande S, Sharma R, Borkar M (2016): Tuberculin purified protein derivative immunotherapy in the treatment of viral warts. Indian J Drugs Dermatol., 2:19-23.

16. Singh SK, Mohan A, Gupta AK, Pandey AK (2018): A comparative study between intralesional PPD and vitamin D3 in treatment of viral warts. Int J Res Dermatol., 4(2):197-201.

17.Saoji V, Lade NR, Gadegone R, Bhat A (2016): Immunotherapy using purified protein derivative in the treatment of warts: An open uncontrolled trial. Indian J Dermatol Venereol Leprol., 82:42-6.

18. Leto Md, Santos Júnior GF, Porro AM, Tomimori J (2011): Human papillomavirus infection: etiopathogenesis, molecular biology and clinical manifestations. An Bras Dermatol., 86(2):306-17.

19. Kus S, Ergun T, Gun D, Akin O (2005): Intrlesional tuberculin for treatment of refractory warts. J Eur Acad Dermatol Ven., 19: 503-23.

20. Elela IM, Elshahid AR, Mosbeh AS (2011): Intradermal vs intralesional purified protein derivatives in treatment of warts. Golf J Deramatol Venereol., 18:21-6.

21. Ural A, Arslan S, Ersoz Ş, Değer B (2014): Verruca vulgaris of the tongue: a case report with a literature review. Bosn J Basic Med Sci., 14(3):136-8.

22.22. Eassa BI, Abou-Bakr AA, El-Khalawany MA (2011). Intradermal injection of PPD as a novel approach of immunotherapy in anogenital warts in pregnant women. Dermatol Ther., 24(1):137-43. 